\title{
NODAL DEFORMATIONS OF SINGULARITIES
}

\author{
Jorge A. GONZÁLEZ-RAMÍREZ
}

\begin{abstract}
In this note we study deformations of a plane curve singularity $(C, P)$ to $\delta(C, P)$ nodes. We see that for some types of singularities the method of A'Campo can be carried on using parametric equations. For such singularities we prove that deformations to $\delta$ nodes can be made within the space of curves of the same degree.
\end{abstract}

\section{Introduction}

Let $f(x, y) \in \mathbb{C}\{x, y\}$ be a germ of analytic function having an isolated critical point at $P=(0,0)$ of Milnor number $\mu(f)<\infty$. It is well known that $\mu(f)$ is the maximum number of critical points that a function close to $f$ can have in a neighborhood of $P$ and that in fact there exist deformations $f_{t}(x, y)$ of $f$ possessing exactly $\mu(f)$ critical points for any $t$ near $0([7],[9])$.

If $f(x, y) \in \mathbb{R}\{x, y\}$ is a real irreducible germ, a remarkable result by A'Campo and Gusein-Zade ([1],[6]) asserts that there exist deformations $f_{t}(x, y)$ of $f$ such that $f_{t}$ has $\mu(f)$ non-degenerated real critical points. Such deformations are called real morsifications and their existence is related to the geometry of the level sets $f_{t}(x, y)=\lambda$. In fact, it is known that $f_{t}(x, y)$ can have the same value on at most $\delta=\mu / 2$ critical points. It is therefore enough to find deformations of $f$ such that the curve $f_{t}(x, y)=0$ has $\delta$ double points with two distinct real tangents (real nodes) and $\delta$ bounded regions where $f_{t}$ has either a maximum or a minimum. Gusein-Zade constructs a real morsification of $f(x, y)$ for the case where the germ of the curve $C=\left\{(x, y) \in \mathbb{C}^{2} ; f(x, y)=0\right\}$ around $P=(0,0)$ has a short real parametrization, that is, if the non-zero terms of its parametrization correspond to the characteristic exponents.

2000 Mathematics Subject Classification: 32S30, 14B07, 14H20.

Servicio de Publicaciones. Universidad Complutense. Madrid, 2002 
A'Campo's method is more geometrical since it uses the resolution of the singular point $P \in C$ by means of quadratic transformations. Let us assume that $(C, P)$ has only one real analytic branch and that $n_{0}, n_{1}, \ldots, n_{k}, \ldots$, is the sequence of multiplicities at the singular points throughout the resolution. By means of a sequence of translations in the direction of the exceptional divisor and by contractions one can obtain a deformation of $f$ having only ordinary singular points whose tangents are real and have multiplicities $n_{0}, n_{1}, \ldots, n_{k}, \ldots$ At the point of multiplicity $n_{i}$ there are exactly $n_{i}$ smooth real branches. Arranging all such branches in general position one obtains $\delta=\sum_{i} n_{i}\left(n_{i}-1\right) / 2$ real nodes. These deformations can be derived from the implicit equations of the function at the sequence of singular points. The drawback of this method is that the equation of the final deformation obtained is not explicit. In particular, if $f(x, y)$ is a polynomial of degree $d$, it is not known in general whether or not the real morsification can be obtained by adding monomials of lower degrees. This question has a geometrical interest, since the existence of such deformations implies that the projective curve $\bar{C} \subset \mathbb{P}_{2}$ can be deformed into a projective curve having $\delta$ nodes in a neighborhood of $P$ inside the space of curves of degree $d$.

In the present work - based on [5] - we prove that for a certain type of singularities A'Campo's method can be applied to deform the parametric equations rather than the implicit ones, and consequently one can obtain the explicit equations of such deformations. We also prove that singularities of type $y^{d-1}-x^{d}$ and $y^{2 d-1}-x^{2 d+1}$ can be deformed into real morsifications preserving the degree of the original polynomial.

\section{Partitions}

Definition 2.1. Let $f(x, y)$ be a polynomial such that its associated plane curve

$$
H=\left\{(x, y) \in \mathbb{C}^{2} ; f(x, y)=0\right\} \subset \mathbb{C}^{2}
$$

has an isolated singularity at $(0,0) \in \mathbb{C}^{2}$. Let

$$
B_{\varepsilon}=\left\{(x, y) \in \mathbb{C}^{2} ;|x|^{2}+|y|^{2} \leq \varepsilon^{2}\right\} \quad,
$$


with $\varepsilon>0$ sufficiently small, a Milnor ball for the singularity of $H$ at $(0,0)$. Let $J$ be the disjoint union of $r$ intervals $[0,1]$ and let $D_{\varepsilon}$ be the disk $D_{\varepsilon}=B_{\varepsilon} \cap \mathbb{R}^{2}$. that:

A partition of $D_{\varepsilon}$ in $r$ branches is an immersion $\gamma: J \longrightarrow D_{\varepsilon}$ such

(i) $\gamma(\partial J) \subset \partial D_{\varepsilon} ; \gamma(\stackrel{\circ}{J}) \subset \stackrel{\circ}{D_{\varepsilon}} ; \gamma(J)$ connected.

(ii) $\gamma$ is generic; that is, $\gamma(J)$ has only ordinary double points.

If $\gamma: J \longrightarrow D_{\varepsilon}$ is a partition then the curve $\gamma(J)$ is also called a partition.

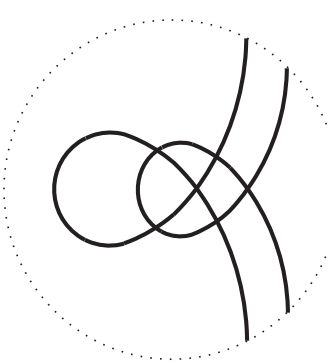

Two-branch partition

Let $f(x, y) \in \mathbb{R}[x, y]$ be a polynomial with an ordinary double point $P_{0}$ of multiplicity $r$-we can also assume $f$ to be regular in $y$ of order $r$, that is, $f(0, y)=y^{r}+$ (higher degree terms in $\left.y\right)$. Let $f_{r}(x, y)=$ $\prod_{j=1}^{r}\left(y-\alpha_{j} x\right)$ be its initial form, then its tangent lines are given by the equations $l_{j} \equiv y-\alpha_{j} x=0, j=1,2, \ldots, r$.

Let us assume there exits a parametrization $\gamma=\left(\gamma_{1}, \gamma_{2}\right): I \longrightarrow \mathbb{R}^{2}$; $\gamma(u)=(x, y)$ such that $\gamma(I) \subset C=\left\{(x, y) \in \mathbb{C}^{2} ; f(x, y)=0\right\} \subset \mathbb{C}^{2}$, that is, $f(\gamma(u)) \equiv 0$ and such that there exist $e_{1}, e_{2}, \ldots, e_{r} \in I$ with $\gamma\left(e_{j}\right)=P_{0}=(0,0)$ and such that the tangent vector $\gamma^{\prime}\left(e_{j}\right)$ is on the line $l_{j}$, for each $j=1,2, \ldots, r$.

Note that this condition about the parametrization is not a direct consequence of the fact that $C$ has a multiple point with $r$ transversal branches. The latter implies the existence of $r$ parametrizations $\delta_{j}$ : 



$I_{j} \longrightarrow \mathbb{R}^{2}$, one for each branch, whereas in our case $\gamma$ parametrizes the $r$ branches simultaneously, producing $r-1$ loops.

Let $\mathbf{v}^{1}, \mathbf{v}^{2}, \ldots, \mathbf{v}^{r} \in \mathbb{R}^{2}-\{(0,0)\}$ be vectors so that the translated lines $\bar{l}_{j}=l_{j}+\mathbf{v}^{j}$ are in general position. The equation of $\bar{l}_{j}$ is $y-v_{2}^{j}-$ $\alpha_{j}\left(x-v_{1}^{j}\right)=0$.

The coordinates of the intersection point, $P_{i k}$, of two lines $\bar{l}_{i}$ and $\bar{l}_{k}$, are

$$
\begin{aligned}
& P_{i k}^{1}=\left(\alpha_{i} v_{1}^{i}-\alpha_{k} v_{1}^{k}-\left(v_{2}^{i}-v_{2}^{k}\right)\right) \frac{1}{\alpha_{i}-\alpha_{k}} \\
& P_{i k}^{2}=\left(\alpha_{i} \alpha_{k}\left(v_{1}^{i}-v_{1}^{k}\right)+\alpha_{i} v_{2}^{k}-\alpha_{k} v_{2}^{i}\right) \frac{1}{\alpha_{i}-\alpha_{k}}
\end{aligned}
$$

Theorem 2.1. Let $f(x, y), \bar{l}_{j}$ and $\mathbf{v}^{j}$ satisfy the previous conditions. Consider $p(u)=\left(p_{1}(u), p_{2}(u)\right)$, where $p_{1}(u), p_{2}(u) \in \mathbb{R}[u]$ are polynomials such that $p\left(e_{j}\right)=\mathbf{v}^{j}, j=1,2, \ldots, d-1$-their existence is assured by taking polynomials of a sufficiently large degree - and let $\bar{\gamma}(u, b)=\gamma(u)+b p(u), b \in \mathbb{R}$.

Then there exists $\varepsilon>0$ such that for any $b$ with $|b|<\varepsilon$, the curve $\bar{\gamma}(u, b)$ is a partition of $r$ branches intersecting generically in $\left(\begin{array}{c}r \\ 2\end{array}\right)$ distinct double points.

Proof. Let us calculate the coordinates of the intersection points of branches $\Gamma_{i}$ and $\Gamma_{k}, i \neq k ; i, k \in\{1,2, \ldots, r\}$; corresponding to $\bar{\gamma}\left(I_{i}\right)$ and $\bar{\gamma}\left(I_{k}\right)$ where $I_{i}=\left[e_{i}-\varepsilon, e_{i}+\varepsilon\right]$ and $I_{k}=\left[e_{k}-\varepsilon, e_{k}+\varepsilon\right]$ are two sufficiently small intervals around $u=e_{i}$ and $u=e_{k}$ respectively.

We will use the following notation $\gamma^{i}\left(u_{i}, b\right)=\bar{\gamma}\left(u_{i}-e_{i}, b\right), \gamma^{k}\left(u_{k}, b\right)=$ $\bar{\gamma}\left(u_{k}-e_{k}, b\right)$ after translating $\bar{\gamma}$ by $u=u_{i}-e_{i}\left(\right.$ resp. $\left.u=u_{k}-e_{k}\right)$ 
in such a way that $\gamma^{i}(0,0)=(0,0), \gamma^{k}(0,0)=(0,0)$, and $\gamma^{i}$ and $\gamma^{k}$ parametrize, respectively, the branches $\Gamma_{i}$ and $\Gamma_{k}$ (i.e. $\gamma^{i}([-\varepsilon, \varepsilon], 0)=\Gamma_{i}$ and $\left.\gamma^{k}([-\varepsilon, \varepsilon], 0)=\Gamma_{k}\right)$.

By the above considerations we know that

$$
\begin{gathered}
\gamma^{i}\left(u_{i}, b\right)=\bar{\gamma}\left(u_{i}-e_{i}, b\right)=\gamma\left(u_{i}-e_{i}\right)+b p\left(u_{i}-e_{i}\right) \\
\gamma^{k}\left(u_{k}, b\right)=\bar{\gamma}\left(u_{k}-e_{k}, b\right)=\gamma\left(u_{k}-e_{k}\right)+b p\left(u_{k}-e_{k}\right) .
\end{gathered}
$$

Moreover, since the tangent lines of $\gamma^{i}$ and $\gamma^{k}$ are $\alpha_{i} x-y=0$ and $\alpha_{k} x-y=0$, respectively, the first terms in the Taylor expansion of $\gamma^{i}$ and $\gamma^{k}$ around $(0,0)$ with respect to the variables $\left(u_{i}, b\right)\left(\operatorname{resp} .\left(u_{k}, b\right)\right)$ are of the form:

$\gamma^{i}\left(u_{i}, b\right)=\left(u_{i}+v_{1}^{i} b+\right.$ higher deg terms, $\alpha_{i} u_{i}+v_{2}^{i} b+$ higher deg terms $)$ $\gamma^{k}\left(u_{k}, b\right)=\left(u_{k}+v_{1}^{k} b+\right.$ higher deg terms, $\alpha_{k} u_{k}+v_{2}^{k} b+$ higher deg terms $)$

Let us calculate the coordinates of a point of intersection of $\Gamma_{i}$ and $\Gamma_{k}$. In order to do so we have to solve the following two equations: $\gamma^{i}\left(u_{i}, b\right)-\gamma^{k}\left(u_{k}, b\right)=(0,0)$.

We define the following function $\psi: \mathbb{R}^{3} \longrightarrow \mathbb{R}^{2}$ as $\psi\left(u_{i}, u_{k}, b\right)=$ $\gamma^{i}\left(u_{i}, b\right)-\gamma^{k}\left(u_{k}, b\right)$. Note that its jacobian matrix at $(0,0,0)$ has the form:

$$
\left(\begin{array}{ccc}
1 & -1 & \left(v_{1}^{i}-v_{1}^{k}\right) \\
\alpha_{i} & -\alpha_{k} & \left(v_{2}^{i}-v_{2}^{k}\right)
\end{array}\right)
$$

This function verifies the following:

There exists an open neighborhood $U \subset \mathbb{R}^{3}$ of $\underline{0}=(0,0,0)$ such that:

(a) $\psi(0,0,0)=\gamma^{i}(0,0)-\gamma^{k}(0,0)=(0,0)$

(b) $\psi$ is of class $C^{\infty}$ on $U$

(c) $\operatorname{det} M=\left|\begin{array}{ll}\frac{\partial \psi^{1}}{\partial u_{i}}(\underline{0}) & \frac{\partial \psi^{1}}{\partial u_{k}}(\underline{0}) \\ \frac{\partial \psi^{2}}{\partial u_{i}}(\underline{0}) & \frac{\partial \psi^{2}}{\partial u_{k}}(\underline{0})\end{array}\right|=\left|\begin{array}{cc}1 & -1 \\ \alpha_{i} & -\alpha_{k}\end{array}\right|=\alpha_{i}-\alpha_{k} \neq 0$ 
Therefore, $\psi\left(u_{i}, u_{k}, b\right)$ satisfies the conditions of the Implicit Function Theorem and thus there exist at least two open neighborhoods $V \subset \mathbb{R}$ and $W \subset \mathbb{R}^{2}$ and a function $\varphi(b)$, such that, for any $b \in V$, there is a unique pair $\left(u_{i}(b), u_{k}(b)\right) \in W$ satisfying $\psi\left(u_{i}(b), u_{k}(b), b\right)=0$. In other words, the function $\varphi: V \longrightarrow W$, defined by $\varphi(b)=\left(u_{i}(b), u_{k}(b)\right)$ (Implicit Function) is differentiable in $V$ and it is the only differentiable function that satisfies $\left\{\left(u_{i}, u_{k}, b\right) \in W \times V ; \psi\left(u_{i}, u_{k}, b\right)=0\right\}=$ $\left\{\left(u_{i}, u_{k}, b\right) \in W \times V ;\left(u_{i}, u_{k}\right)=\varphi(b)\right\}$. Hence, $\varphi(b)=\left(u_{i}(b), u_{k}(b)\right)$ is the solution to both equations: $\psi\left(u_{i}(b), u_{k}(b), b\right)=0$.

Let us calculate the expressions of $u_{i}(b)$ and $u_{k}(b)$.

The derivatives at $0,\left(d u_{i} / d b\right)(0)$ and $\left(d u_{k} / d b\right)(0)$ can be obtained knowing that

$$
\gamma^{i}\left(u_{i}(b), b\right)-\gamma^{k}\left(u_{k}(b), b\right) \equiv(0,0)
$$

Derivating this expression with respect to $b$ and evaluating at 0 results in a system whose only solution is:

$$
\begin{aligned}
& \frac{d u_{i}}{d b}(0)=\frac{1}{\alpha_{i}-\alpha_{k}}\left(\alpha_{k}\left(v_{1}^{i}-v_{1}^{k}\right)-\left(v_{2}^{i}-v_{2}^{k}\right)\right) \\
& \frac{d u_{k}}{d b}(0)=\frac{1}{\alpha_{i}-\alpha_{k}}\left(\alpha_{i}\left(v_{1}^{i}-v_{1}^{k}\right)-\left(v_{2}^{i}-v_{2}^{k}\right)\right)
\end{aligned}
$$

Thus, $u_{i}(b)$ and $u_{k}(b)$ have the form

$$
\begin{aligned}
& u_{i}(b)=\frac{1}{\alpha_{i}-\alpha_{k}}\left(\alpha_{k}\left(v_{1}^{i}-v_{1}^{k}\right)-\left(v_{2}^{i}-v_{2}^{k}\right)\right) b+(\text { higher degree terms in } b) \\
& u_{k}(b)=\frac{1}{\alpha_{i}-\alpha_{k}}\left(\alpha_{i}\left(v_{1}^{i}-v_{1}^{k}\right)-\left(v_{2}^{i}-v_{2}^{k}\right)\right) b+(\text { higher degree terms in } b)
\end{aligned}
$$

Therefore, the double point which is intersection of the branches $\Gamma_{i}$ and $\Gamma_{k}$ has coordinates

$$
\omega^{i k}(b)=\gamma^{i}\left(u_{i}(b), b\right)=\gamma^{k}\left(u_{k}(b), b\right)=\left(\omega_{1}^{i k}, \omega_{2}^{i k}\right) .
$$

Substituting (3) in (2) one obtains that

$$
\begin{aligned}
& \omega_{1}^{i k}=\left(\alpha_{i} v_{1}^{i}-\alpha_{k} v_{1}^{k}-\left(v_{2}^{i}-v_{2}^{k}\right)\right) \frac{1}{\alpha_{i}-\alpha_{k}} b+(\text { higher degree terms in } b) \\
& \omega_{2}^{i k}=\left(\alpha_{i} \alpha_{k}\left(v_{1}^{i}-v_{1}^{k}\right)+\alpha_{i} v_{2}^{k}-\alpha_{k} v_{2}^{i}\right) \frac{1}{\alpha_{i}-\alpha_{k}} b+(\text { higher deg terms in } b)
\end{aligned}
$$


Let $\omega^{i k}(b)$ be the intersection point of the branches $\Gamma_{i}$ and $\Gamma_{k}$. Consider two pairs of branches $\left\{\Gamma_{i}, \Gamma_{k}\right\}$ and $\left\{\Gamma_{i^{\prime}}, \Gamma_{k^{\prime}}\right\}$ having at least a noncommon element. In order to show that $\omega^{i k}(b)$ and $\omega^{i^{\prime} k^{\prime}}(b)$ are different for $b$ sufficiently small, it is enough to check that the curves defining such double points, say $\omega^{i k}$ and $\omega^{i^{\prime} k^{\prime}}$ have different derivatives at 0 . As it is easily seen, the derivative $\underline{0}$ of $\omega^{i k}$, verifies

$$
\frac{d \omega^{i k}}{d b}(\underline{0})=P_{i k} \quad \text { and } \quad P_{i k} \neq P_{i^{\prime} k^{\prime}} \quad(\text { see }(2.1)) .
$$

Therefore, the curve $\bar{\gamma}(u, b)$ is a partition of $r$ branches intersecting in $\left(\begin{array}{l}r \\ 2\end{array}\right)$ distinct double points.

Theorem 2.2. Under the conditions of the previous theorem (2.1), for any $b$ sufficiently small, the $r$ branches of $\bar{\gamma}(u, b)$ produce a partition of the same real combinatorial type as the $r$ lines $\bar{l}_{j}, j=1,2, \ldots, r$ (translated of $l_{j}$, by the vector $\mathbf{v}^{j}$ ).

Proof. Let $\bar{l}_{j}^{b}=l_{j}+b \mathbf{v}^{j}$ be the line $l_{j}$ translated by the vector $b \mathbf{v}^{j}=$ $\left(b v_{1}^{j}, b v_{2}^{j}\right)$. Note that the line $\bar{l}_{j}$ is $\left.\bar{l}_{j}^{1}(b=1)\right)$.

Let us calculate the coordinates of the intersection points of the translated lines $\bar{l}_{i}^{b}$ and $\bar{l}_{k}^{b}, i \neq k ; i, k \in\{1,2, \ldots, r\}$.

Solving the system formed by the equations of both lines

$$
\left.\begin{array}{l}
\bar{l}_{i}^{b} \equiv y-b v_{2}^{i}-\alpha_{i}\left(x-b v_{1}^{i}\right)=0 \\
\bar{l}_{k}^{b} \equiv y-b v_{2}^{k}-\alpha_{k}\left(x-b v_{1}^{k}\right)=0
\end{array}\right\}
$$

it turns out that their intersection point, $P_{i k}^{b}$, has the following coordinates:

$$
\begin{gathered}
P_{i k}^{b 1}=\left(\alpha_{i} v_{1}^{i}-\alpha_{k} v_{1}^{k}-\left(v_{2}^{i}-v_{2}^{k}\right)\right) \frac{1}{\alpha_{i}-\alpha_{k}} b \\
P_{i k}^{b 2}=\left(\alpha_{i} \alpha_{k}\left(v_{1}^{i}-v_{1}^{k}\right)+\alpha_{i} v_{2}^{k}-\alpha_{k} v_{2}^{i}\right) \frac{1}{\alpha_{i}-\alpha_{k}} b
\end{gathered}
$$

It is clear that the arrangement of double points of $\cup_{j=1}^{r} \bar{l}_{j}^{b}$ comes from a dilation of ratio $b$ from $\cup_{j=1}^{r} \bar{l}_{j}$. On the other hand, by the proof of the previous theorem (2.1), the analytical expression of the intersection point of the branches $\Gamma_{i}$ and $\Gamma_{k}$ is

$$
\omega^{i k}(b)=P_{i k}^{b}+(\text { terms of degree } \geq 2 \text { in } b) .
$$


Therefore it is obvious that the distance between the points $\omega^{i k}(b)$ and $P_{i k}^{b}$ is of order $b^{2}$, whereas the distance between the points of intersection $P_{i k}^{b}$ and $P_{i^{\prime} k^{\prime}}^{b}$ is of order $b$. Thus, for sufficiently small values of $b$, the points $P_{i k}^{b}$ and $\omega^{i k}(b)$ are very close, and their difference at $b$ starts with terms of degree greater or equal to 2 in terms of the coordinates of $b$. This implies the same real combinatorial type of both arrangements.



Finally, note that the $r-1$ intersection points of a branch with the remaining $r-1$ branches correspond to the values of $\omega^{i k}(b)$, with $k \neq i$, where $u_{i}$ are close to 0 . In the original parametrization this translates into values of $u$ close to $e_{i}$. That is, the parametrization runs over the $r-1$ intersection points in $\Gamma_{i}$ before finishing a loop and starting the next one.

Theorem 2.3. Let $m, n \in \mathbb{N}, m>n,(m, n)=1 ; \bar{\gamma}_{\underline{t}}(u)=\left(\bar{\gamma}_{\underline{\underline{t}}}^{1}(u), \bar{\gamma}_{\underline{\underline{t}}}^{2}(u)\right)$ where

$$
\bar{\gamma}_{\underline{t}}^{1}(u)=u^{n}+\sum_{j=1}^{n} a_{j}(t) u^{n-j} \quad, \quad \bar{\gamma}_{\underline{t}}^{2}(u)=u^{m}+\sum_{j=1}^{m} b_{j}(t) u^{m-j}
$$

$\underline{t} \in \mathbb{R}^{e}, a_{j}(\underline{0})=0$ and $b_{j}(\underline{0})=0$ and let

$$
F(x, y ; \underline{t})=\operatorname{Res}_{u}\left(\bar{\gamma}_{\underline{t}}^{1}(u)-x, \bar{\gamma}_{\underline{t}}^{2}(u)-y\right) .
$$

Then, $F(x, y ; \underline{t})$ is a polynomial function of degree $m$ in the variables $x$ and $y$, and $F(x, y ; \underline{0})=y^{n}-x^{m}$. 
Proof. Since $\bar{\gamma}_{\underline{\underline{t}}}^{1}(u)-x$ and $\bar{\gamma}_{\underline{\underline{t}}}^{2}(u)$ are both of the form:

$$
\begin{aligned}
& \bar{\gamma}_{\underline{t}}^{1}(u)-x=u^{n}+\left(\sum_{j=1}^{n} a_{j}(t) u^{n-j}\right)-x \\
& \bar{\gamma}_{\underline{\underline{t}}}^{2}(u)-y=u^{m}+\left(\sum_{j=1}^{m} b_{j}(t) u^{m-j}\right)-y
\end{aligned}
$$

they are monic in the variable $u$. Hence

$$
\operatorname{Im}\left(\bar{\gamma}_{\underline{\underline{t}}}(u)\right)=\{(x, y ; \underline{t}) ; F(x, y ; \underline{t})=0\}
$$

\begin{tabular}{|c|c|c|c|c|c|c|c|c|c|}
\hline 1 & $a_{1}$ & $a_{2}$ & $\ldots \ldots$ & $a_{n-1}$ & $a_{n}-x$ & 0 & & & 0 \\
\hline 0 & 1 & $a_{1}$ & $a_{2}$ & $\ldots \ldots$ & $a_{n-1}$ & $a_{n}-x$ & 0 & $\cdots$ & 0 \\
\hline 0 & .. & . & .. & 0 & 1 & $a_{1}$ & $a_{2}$ & $a_{n-1}$ & $a_{n}-x$ \\
\hline 1 & $b_{1}$ & $b_{2}$ & $\ldots$ & $b_{m-2}$ & $b_{m-1}$ & $b_{m}-y$ & 0 & $\ldots \ldots \ldots$ & 0 \\
\hline 0 & 1 & $b_{1}$ & $b_{2}$ & . & $b_{m-2}$ & $b_{m-1}$ & $b_{m}-y$ & 0 & 0 \\
\hline
\end{tabular}

The implicit equation of $F(x, y ; \underline{t})$ is given by the determinant of the Sylvester $n+m$ matrix:

By a direct analysis of this matrix, one sees that the only non-constant polynomials in $x$ and $y$ belong to the rows $n+1, n+2, \ldots, n+m$ and are linear polynomials in $x$ and $y$. All the elements in the remaining rows do not depend on $x$ or $y$. Therefore, when computing the determinant, $m$ is an upper bound of its degree in the variables $x$ and $y$.

On the other hand, if $\underline{t}=\underline{0}$, then $a_{j}=0$ and $b_{j}=0$ for every $j=1,2, \ldots, m$. Therefore it follows that $F(x, y ; \underline{0})=y^{n}-x^{m}$.

\section{Type $y^{d-1}-x^{d}$ singularities}

Proposition 3.1. Let $f(x, y)=y^{d-1}-x^{d} \in \mathbb{R}[x, y]$ be a polynomial where $d \leq 3$. The real polynomial

$$
f(x, y ; t)=\prod_{j=1}^{d-1}\left(y-\alpha_{j} t x\right)-x^{d}
$$


with $t, \alpha_{j} \in \mathbb{R}-\{0\} ; j=1,2, \ldots, d-1$ and $\alpha_{k} \neq \alpha_{i}$ if $k \neq i$; is a deformation of $f(x, y)$. Moreover, the plane curve

$$
C_{t}=\left\{(x, y) \in \mathbb{C}^{2} ; f(x, y ; t)=0\right\} \subset \mathbb{C}^{2}
$$

has a unique ordinary singular point at $(0,0)$ of multiplicity $d-1$.

Proof. The plane curve $H=\left\{(x, y) \in \mathbb{C}^{2} ; f(x, y)=0\right\} \subset \mathbb{C}^{2}$ has an isolated singularity at $(0,0)$. The initial form of $f(x, y ; t), \prod_{j=1}^{d-1}\left(y-\alpha_{j} t x\right)$, has degree $d-1$ and $d-1$ distinct roots: $y=\alpha_{d-1} t x ; y=\alpha_{d-2} t x ; \ldots$; $y=\alpha_{2} t x ; y=\alpha_{1} t x$.

Hence, for any generic value of $t \neq 0$ and $\alpha_{k} \neq \alpha_{i}$ for $k \neq i$, one obtains $d-1$ distinct tangents at the origen.

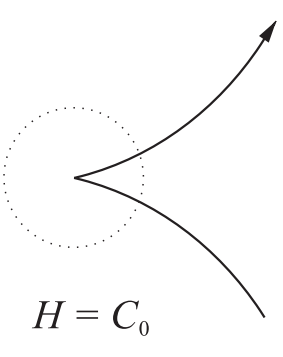

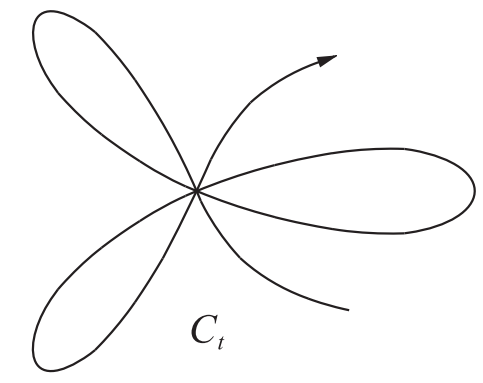

Case $d=5$

In Theorem (2.1), it was described how to deform a parametrization $\gamma(u)$ of a curve $C \equiv f(x, y)=0$, with one ordinary singular point of multiplicity $r$, in such a way that $\left(\begin{array}{l}r \\ 2\end{array}\right)$ new nodes are produced. Something similar will be performed to a parametrization of the family $C_{t}$ which - as we just proved - has one ordinary singular point for each $t \neq 0$. The main difficulty in this case is to check that the required functions are actually analytical for $t \neq 0$, and have no poles for $t=0$.

Let $\gamma: J \times I \longrightarrow \mathbb{R}^{2}$ be a mapping such that, for any $t \neq 0$, $\gamma_{t}(u)=\gamma(u, t)$ is a parametrization of $C_{t}$ satisfying the following additional property: if we denote by $e_{1}(t), e_{2}(t), \ldots, e_{d-1}(t)$, the parameters of $u$ for which $\gamma_{t}\left(e_{j}(t)\right)=(0,0)$, then we ask these parameters to satisfy $e_{j}(t)=e_{j} t$, where $e_{j} \in \mathbb{R}$. It is easy to see that such a parametrization exists.

Let $l_{j} \equiv \alpha_{j} t x-y=0, j=1,2, \ldots d-1$ be the tangent lines of $C_{t}$ at the origin, and let $\mathbf{v}^{j}=\left(v_{1}^{j} t^{d-1}, v_{2}^{j} t^{d-1}\right) \in \mathbb{R}^{2}, j=1,2, \ldots, d-1$ 
be vectors such that the translated lines $\bar{l}_{j}=l_{j}+\mathbf{v}^{j}$, intersect to each other in distinct points. Hence, $\bar{l}_{j} \equiv \alpha_{j} t\left(x-v_{1}^{j} t^{d-1}\right)-\left(y-v_{2}^{j} t^{d-1}\right)$, $j=1,2, \ldots, d-1$ and the intersection point of two lines $\bar{l}_{i}$ and $\bar{l}_{k}$ has coordinates of the form:

$$
\begin{aligned}
& P_{i k}^{1}=\left(\alpha_{i} v_{1}^{i}-\alpha_{k} v_{1}^{k}-\left(v_{2}^{i}-v_{2}^{k}\right)\right) \frac{t^{d-2}}{\alpha_{i}-\alpha_{k}} \\
& P_{i k}^{2}=\left(\alpha_{i} \alpha_{k}\left(v_{1}^{i}-v_{1}^{k}\right)+\alpha_{i} v_{2}^{k}-\alpha_{k} v_{2}^{i}\right) \frac{t^{d-1}}{\alpha_{i}-\alpha_{k}}
\end{aligned}
$$

Let $p_{t}(u)=\left(p_{1}(u, t), p_{2}(u, t)\right)$ where $p_{1}(u, t), p_{2}(u, t)$

$\in \mathbb{R}[u, t]$ are polynomials such that $p_{t}\left(e_{j} t\right)=\left(v_{1}^{j} t^{d-2}, v_{2}^{j} t^{d-2}\right), j=$ $1,2, \ldots, d-1$. Such polynomials exist for large enough degrees. For example,

$$
p_{1}(u, t)=\sum_{j=1}^{d-1} v_{1}^{j} L_{j}(u, t) \quad \text { and } \quad p_{2}(u, t)=\sum_{j=1}^{d-1} v_{2}^{j} L_{j}(u, t)
$$

with

$$
L_{j}(u, t)=\prod_{\substack{k=1 \\ k \neq j}}^{d-1} \frac{\left(u-e_{k} t\right)}{\left(e_{j}-e_{k}\right)}
$$

Proposition 3.2. Under the above conditions, let $\bar{\gamma}(u, b, t)=\gamma_{t}(u)+$ $b t p_{t}(u), b \in \mathbb{R}-\{0\}$. Then, for any $b$ and $t$ sufficiently small, the curve $\bar{\gamma}(u, b, t)$ is a partition of $d-1$ branches intersecting generically in $\left(\begin{array}{c}d-1 \\ 2\end{array}\right)$ distinct double points.

Proof. It basically follows the proof of Theorem (2.1) in the previous section. The only difference being that the parametrization required in this proof also depends on $t$. We will only stress the points of divergence with the aforementioned proof.

By Lagrange interpolation formula, a polynomial $p$ satisfying $p\left(u_{1}\right)=$ $w_{1}, p\left(u_{2}\right)=w_{2}, \ldots, p\left(u_{d-1}\right)=w_{d-1}$ has the form

$$
p(u)=\sum_{j=1}^{d-1} w_{j} \bar{L}_{j}(u) \quad \text { where } \bar{L}_{j}(u)=\prod_{\substack{k=1 \\ k \neq j}}^{d-1} \frac{\left(u-u_{k}\right)}{\left(u_{j}-u_{k}\right)}
$$


In our case we have $u_{j}=e_{j} t$, for each $j=1,2, \ldots, d-1$. Also the denominator of $\bar{L}_{j}(u)$ looks as follows $t^{d-2} \prod_{k=1}^{d-1}\left(e_{j}-e_{k}\right)$. Therefore: $k \neq j$

$$
\bar{L}_{j}(u)=\frac{1}{t^{d-2}} L_{j}(u, t) \quad \text { where } L_{j}(u, t)=\prod_{\substack{k=1 \\ k \neq j}}^{d-1} \frac{\left(u-e_{k} t\right)}{\left(e_{j}-e_{k}\right)}
$$

Considering $w_{j}=v_{1}^{j} t^{d-2}$ (resp. $\left.w_{j}=v_{2}^{j} t^{d-2}\right)$ we have

$$
p_{1}(u, t)=\sum_{j=1}^{d-1} v_{1}^{j} L_{j}(u, t) \quad\left(\text { resp. } p_{2}(u, t)=\sum_{j=1}^{d-1} v_{2}^{j} L_{j}(u, t)\right) .
$$

As in Theorem (2.1), we will calculate the coordinates of the intersection points of the branches $\Gamma_{i}$ and $\Gamma_{k}(i \neq k ; 1, k \in\{1,2, \ldots, d-1\})$, corresponding to $\bar{\gamma}_{t}\left(I_{i}\right)$ and $\bar{\gamma}_{t}\left(I_{k}\right)$, where $I_{i}=\left[e_{i} t-\varepsilon, e_{i} t+\varepsilon\right]$ and $I_{k}=\left[e_{k} t-\varepsilon, e_{k} t+\varepsilon\right]$ are two sufficiently small intervals around $u=e_{i} t$ and $u=e_{k} t$ respectively.

Denoting $\gamma^{i}\left(u_{i}, b, t\right)$ by $\bar{\gamma}\left(u_{i}-e_{i} t, b, t\right)\left(\right.$ resp. $\gamma^{k}\left(u_{k}, b, t\right)$ by $\bar{\gamma}\left(u_{k}-\right.$ $\left.\left.e_{k} t, b, t\right)\right)$ one has $\gamma^{i}\left(u_{i}, b, t\right)=\gamma_{t}\left(u_{i}-e_{i} t\right)+b t p_{t}\left(u_{i}-e_{i} t\right) ; \gamma^{k}\left(u_{k}, b, t\right)=$ $\gamma_{t}\left(u_{k}-e_{k} t\right)+b t p_{t}\left(u_{k}-e_{k} t\right)$. Since the tangent lines of $\gamma^{i}$ and $\gamma^{k}$ at the origin are $\alpha_{i} t x-y=0$ and $\alpha_{k} t x-y=0$, respectively, the first terms in the Taylor expansions of $\gamma^{i}$ and $\gamma^{k}$ at $(0,0)$ have the form:

$$
\begin{aligned}
& \gamma^{i}\left(u_{i}, b, t\right)= \\
& \left(u_{i}+v_{1}^{i} b t^{d-1}+\text { higher deg terms, } \alpha_{i} t u_{i}+v_{2}^{i} b t^{d-1}+\text { higher deg terms }\right) \\
& \gamma^{k}\left(u_{k}, b, t\right)= \\
& \left(u_{k}+v_{1}^{k} b t^{d-1}+\text { higher deg terms, } \alpha_{k} t u_{k}+v_{2}^{k} b t^{d-1}+\text { higher deg terms }\right)
\end{aligned}
$$

In order to solve the two equations: $\gamma^{i}\left(u_{i}, b, t\right)-\gamma^{k}\left(u_{k}, b, t\right)=(0,0)$ we define the function $\psi: \mathbb{R}^{4} \longrightarrow \mathbb{R}^{2} ; \psi\left(u_{i}, u_{k}, b, t\right)=\gamma^{i}\left(u_{i}, b, t\right)-$ $\gamma^{k}\left(u_{k}, b, t\right)$. This function verifies the hypothesis of the Implicit Function Theorem in an open neighborhood of $O=(0,0,0, t), t \neq 0$. (Note that, in this case, the jacobian matrix of $\psi$ at $O$ has a zero column and that 
the third hypothesis is a consequence of the fact that $\left(\alpha_{i}-\alpha_{k}\right) t \neq 0$ since $t \neq 0)$. Hence, the implicit function $\varphi(b, t)=\left(u_{i}(b, t), u_{k}(b, t)\right)$ is the solution to the aforementioned equations. Calculating the derivative with respect to $b$ for the expression $\gamma^{i}\left(u_{i}(b), b, t\right)-\gamma^{k}\left(u_{k}(b), b, t\right) \equiv(0,0)$ and evaluating at $(0, t)$ one obtains a system of equations whose only solution is

$$
\begin{aligned}
& \frac{\partial u_{i}}{\partial b}(0, t)=\frac{t^{d-2}}{\alpha_{i}-\alpha_{k}}\left(\alpha_{k} t\left(v_{1}^{i}-v_{1}^{k}\right)-\left(v_{2}^{i}-v_{2}^{k}\right)\right) \\
& \frac{\partial u_{k}}{\partial b}(0, t)=\frac{t^{d-2}}{\alpha_{i}-\alpha_{k}}\left(\alpha_{i} t\left(v_{1}^{i}-v_{1}^{k}\right)-\left(v_{2}^{i}-v_{2}^{k}\right)\right)
\end{aligned}
$$

Hence, $u_{i}(b, t)$ and $u_{k}(b, t)$ become

$$
\begin{aligned}
& u_{i}(b, t)=\frac{t^{d-2}}{\alpha_{i}-\alpha_{k}}\left(\alpha_{k} t\left(v_{1}^{i}-v_{1}^{k}\right)-\left(v_{2}^{i}-v_{2}^{k}\right)\right) b+(\text { higher deg terms in } b) \\
& u_{k}(b, t)=\frac{t^{d-2}}{\alpha_{i}-\alpha_{k}}\left(\alpha_{i} t\left(v_{1}^{i}-v_{1}^{k}\right)-\left(v_{2}^{i}-v_{2}^{k}\right)\right) b+(\text { higher deg terms in } b)
\end{aligned}
$$

Hence, substituting (3.3) in (3.2), one obtains the double point $\omega^{i k}$, which is the intersection of the branches $\Gamma_{i}$ and $\Gamma_{k}$. Its coordinates are:

$$
\begin{aligned}
& \omega_{1}^{i k}=\left(\left(\alpha_{i} v_{1}^{i}-\alpha_{k} v_{1}^{k}\right) t-\left(v_{2}^{i}-v_{2}^{k}\right)\right) \frac{t^{d-2}}{\alpha_{i}-\alpha_{k}} b+(\text { higher deg terms in } b) \\
& \omega_{2}^{i k}=\left(\alpha_{i} \alpha_{k}\left(v_{1}^{i}-v_{1}^{k}\right) t+\alpha_{i} v_{2}^{k}-\alpha_{k} v_{2}^{i}\right) \frac{t^{d-1}}{\alpha_{i}-\alpha_{k}} b+(\text { higher deg terms in } b)
\end{aligned}
$$

Finally, it remains to check that $\omega^{i k}(b, t)$ is different from $\omega^{i^{\prime} k^{\prime}}(b)$ for any two pairs of branches $\left\{\Gamma_{i}, \Gamma_{k}\right\}$ and $\left\{\Gamma_{i^{\prime}}, \Gamma_{k^{\prime}}\right\}$ having at least a non-common point and for any $t$ and $b$ sufficiently small. In order to do so, it is enough to check that the curves defining $\omega^{i k}$ and $\omega^{i^{\prime} k^{\prime}}$ have different derivatives at with respect to $b$ at $(0, t)$. This is a consequence of the following fact

$$
\frac{\partial \omega^{i k}}{\partial b}(0, t)=P_{i k} \quad \text { and } \quad P_{i k} \neq P_{i^{\prime} k^{\prime}} \quad(\text { see }(3.1)) .
$$

Hence, the curve $\bar{\gamma}(u, b, t)$ is a partition of $d-1$ branches intersecting to each other generically in $\left(\begin{array}{c}d-1 \\ 2\end{array}\right)$ distinct double points.

Proposition 3.3. Under the hypothesis of the previous proposition (3.2), the branches of $\bar{\gamma}(u, b, t)$ produce a partition whose real combinatorics coincide with the one of the arrangement of translated lines $\bar{l}_{j}=l_{j}+\mathbf{v}^{j}, j=1,2, \ldots, d-1$. 


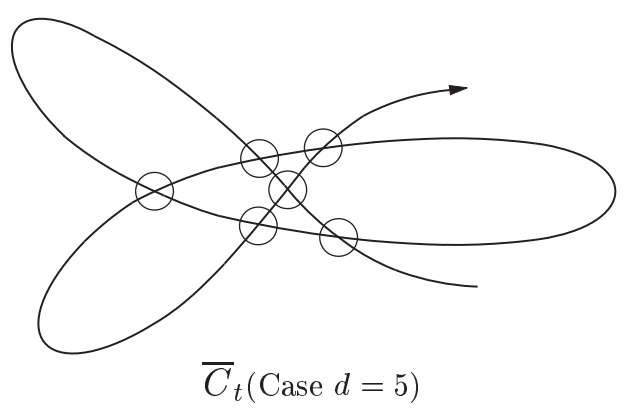

Proof. The result follows by using Theorem(1.2) with the hypothesis of Proposition (3.2).

Remark. In Proposition (2.2), the curve $\bar{\gamma}(u, b, t)=\gamma_{t}(u)+b t p_{t}(u)$ depends on the values of the parameters $b$ and $t$.

From now on we will consider

$$
\gamma_{t}(u)=\left(\prod_{j=1}^{d-1}\left(u-\alpha_{j} t\right), u \prod_{j=1}^{d-1}\left(u-\alpha_{j} t\right)\right)
$$

as the parametrization of

$$
C_{t}=\left\{(x, y) \in \mathbb{C}^{2} ; f(x, y ; t)=\prod_{j=1}^{d-1}\left(y-\alpha_{j} t x\right)-x^{d}=0\right\} .
$$

This parametrization satisfies the hypothesis of Proposition (2.2). Moreover, since $e_{j}=\alpha_{j}$, we have $\gamma_{t}\left(\alpha_{j} t\right)=(0,0)$, for $j=1,2, \ldots, d-1$.

Let $\gamma_{t}(u)$ be the parametrization and let $\bar{\gamma}(u, b, t)$ be the deformation constructed in Proposition (3.2). That is,

$$
\bar{\gamma}(u, b, t)=\gamma_{t}(u)+b t p_{t}(u)=\left(\bar{\gamma}_{t}^{1}(u, b), \bar{\gamma}_{t}^{2}(u, b)\right),
$$

where

$$
\bar{\gamma}_{t}^{1}(u, b)=\prod_{j=1}^{d-1}\left(u-\alpha_{j} t\right)+b t \sum_{j=1}^{d-1} v_{1}^{j} \prod_{\substack{k=1 \\ k \neq j}}^{d-1} \frac{\left(u-\alpha_{k} t\right)}{\left(\alpha_{j}-\alpha_{k}\right)}
$$




$$
\bar{\gamma}_{t}^{2}(u, b)=u \prod_{j=1}^{d-1}\left(u-\alpha_{j} t\right)+b t \sum_{j=1}^{d-1} v_{2}^{j} \prod_{\substack{k=1 \\ k \neq j}}^{d-1} \frac{\left(u-\alpha_{k} t\right)}{\left(\alpha_{j}-\alpha_{k}\right)}
$$

Proposition 3.4. Let $\bar{f}(x, y, b, t)=\operatorname{Res}_{u}\left(\bar{\gamma}_{t}^{1}(u, b)-x, \bar{\gamma}_{t}^{2}(u, b)-y\right)$. Then, $\bar{f}(x, y, b, t)$ is a degree $d$ polynomial in the variables $x$ and $y$ satisfying $\bar{f}(x, y, 0,0)=y^{d-1}-x^{d}$.

Proof. First note that both $\bar{\gamma}_{t}^{1}(u, b)$ and $\bar{\gamma}_{t}^{2}(u, b)$ have the following form:

$$
\begin{aligned}
& \bar{\gamma}_{t}^{1}(u, b)=u^{d-1}+a_{1} u^{d-2}+a_{2} u^{d-3}+\ldots+a_{d-2} u+a_{d-1} \\
& \bar{\gamma}_{t}^{2}(u, b)=u^{d}+b_{1} u^{d-1}+b_{2} u^{d-2}+\ldots+b_{d-2} u^{2}+b_{d-1} u+b_{d}
\end{aligned}
$$

where the coefficients $a_{j}$ and $b_{j}, j=1,2, \ldots, d$, are polynomials in the variables $b$ and $t$ without degree zero terms in $t$. The result follows by reproducing the proof of Theorem (2.3) and will be omitted.

Let $D \subset \mathbb{C}^{2}$ be a nodal curve. Changing coordinates if necessary, we can assume that no line in the pencil $x=$ constant is tangent to $D$ at an inflexion point and that any of such lines contains at most one node of $D$. Let $g(x, y)=0$ be an equation for $D$ in such a coordinate system and let us denote by $\Delta(x)$ the discriminant of $g$ with respect to $y$, $\Delta(x)=\operatorname{Disc}_{y}(g(x, y))$. In the above conditions $\Delta(x)$ has $r$ double roots corresponding to the nodes of $D$ and the rest of his roots are simple, corresponding to the projection of an smooth points of $D$ whose tangent is vertical. Let $R(x)=\operatorname{gcd}\left(D(x), D_{x}(x)\right)$, then $R(x)$ has degree $r$ in $x$, because its roots are the double roots of $D(x)$. By using the principal resultants we can expres the last condition as follows:

Let $M$ the Sylvester matrix whose determinant gives $D i s c_{x}(D(x))$, and $M_{j}$ the submatrix of $M$ formed by deleting the last $j$ rows of terms from $D(x)$, the last $j$ rows of terms from $D_{x}(x)$ and the last $2 j$ columns. Then we have (see [3] Thm. 7.3, pg. 289) :

$$
\begin{gathered}
\operatorname{degree}_{\left(\operatorname{Disc}_{x}(D(x))=r \quad\right. \text { if and only }} \\
\operatorname{det}\left(M_{0}\right)=\ldots=\operatorname{det}\left(M_{r-1}\right)=0 \text { and } \operatorname{det}\left(M_{r}\right) \neq 0
\end{gathered}
$$

Theorem 3.1. Under the previous conditions let $U$ be the following set 


$$
U=\left\{(b, t) \in \mathbb{C}^{2} ; \bar{\gamma}(u, b, t) \text { has }\left(\begin{array}{c}
d-1 \\
2
\end{array}\right) \text { distinct nodes }\right\} .
$$

Then, $U$ contains a dense Zariski open set and, therefore, there exists an analitic curve $\beta: J \longrightarrow \mathbb{C}^{2} ; \beta(s)=(b(s), t(s))$ so that $\beta(J-\{0\}) \subset U$. In other words, $\tilde{\gamma}(u, s)=\bar{\gamma}(u, b(s), t(s))=\bar{\gamma}(u, \beta(s))$ has $\left(\begin{array}{c}d-1 \\ 2\end{array}\right)$ distinct double points for any $s \neq 0$.

Proof. Let $\bar{f}(x, y, b, t)$ be the deformation of $f(x, y)=y^{d-1}-x^{d}$ obtained in Proposition (3.4) and let take $\left(b_{0}, t_{0}\right)$ so that $\bar{f}\left(x, y, b_{0}, t_{0}\right)=0$ is a nodal curve with $r=\left(\begin{array}{c}d-1 \\ 2\end{array}\right)$. Let $\Delta(x, b, t)=\operatorname{Disc}_{y}(\bar{f}(x, y, b, t)) \in$ $\mathbb{R}[x, y, t]$ be the discriminant of $\bar{f}(x, y, b, t)$ with respect to $y$. Then, one can find a coordinate system such that the only multiple roots of $\Delta\left(x, b_{0}, t_{0}\right)$ are $r$ double roots in $x$.

In a neighborhood of $\left(b_{0}, t_{0}\right)$ the curve $\bar{f}(x, y, b, t)=0$ has $r$ nodes. As we are in the situation described above, the principal resultants verify :

$$
\operatorname{det}\left(M_{0}\right)(b, t)=\ldots=\operatorname{det}\left(M_{r-1}\right)(b, t)=0 \quad \text { and } \quad \operatorname{det}\left(M_{r}\right)\left(b_{0}, t_{0}\right) \neq 0
$$

Since $H=\left\{(b, t) \in \mathbb{C}^{2} ; \operatorname{det}\left(M_{r}\right)(b, t)=0\right\}$ is an algebraic curve with a finite number of branches, $\mathbb{C}^{2}-H$ is a Zariski open dense set and $U \supset \mathbb{C}^{2}-H$. Thus, there are infinitely many analytic branches $\beta: J \longrightarrow U$ such that $\beta(J-\{0\}) \subset U$.

Theorem 3.2. Let $f(x, y)=y^{d-1}-x^{d} \in \mathbb{C}[x, y]$ with $d \geq 3$.

Then there exist deformations of $f(x, y)$, say $\bar{f}(x, y ; s)$, in degree $d$ such that, for any $s \in \mathbb{R}-\{0\}$ sufficiently small, the plane curve $\bar{C}_{s} \equiv \bar{f}(x, y ; s)=0$ has $\left(\begin{array}{c}d-1 \\ 2\end{array}\right)$ distinct real double points.

Proof. The result is a straightforward consequence of the previous propositions. In particular, the parametrization given in Proposition (3.2) proves that the nodes are real.

A known result [2] can be also deduce from this Theorem.

Corollary 3.1. There exist irreducible curves of degree $d$ having $\left(\begin{array}{c}d-1 \\ 2\end{array}\right)$ distinct real nodes (note that this is the upper bound).

\section{Type $y^{2 d-1}-x^{2 d+1}$ singularities}

Essentially A'Campo's method can be stated as follows: For any given isolated singularity $f(x, y)$, one can obtain a degeneration 
$\bar{f}\left(x, y ; t_{1}, t_{2}, \ldots, t_{k}\right)$ where $k$ is the number of blowing-ups performed in order to obtain an embbeded resolution and each parameter $t_{j}(j=$ $1,2, \ldots, k)$ results in the $(k-j)$-th blow-up after performing a suitable translation. In this context, A'Campo states that $\bar{f}(x, y ; t, t, \ldots, t)$ has $\delta(\bar{f})$ real nodes. We will show a simple example $\left(y^{3}-x^{5}=0\right)$ that proves that this statement is not correct.

After performing the following quadratic transformations $\pi_{1}\left(x_{1}, y_{1}\right)=$ $\left(x_{1}, x_{1} y_{1}\right) ; \pi_{2}\left(x_{2}, y_{2}\right)=\left(x_{2} y_{2}, y_{2}\right) ; \pi_{3}\left(x_{3}, y_{3}\right)=\left(x_{3}, x_{3} y_{3}\right)$, one has:

Considering sufficiently small $t_{1}, t_{2}, t_{3} \in \mathbb{R}-\{0\}$ and performing the following translation $\tau_{3}\left(x_{3}, y_{3}\right)=\left(x_{3}-t_{3}, y_{3}\right)$ in a non-exceptional component, 
and, finally, the contraction $\pi_{1}(x, y)$ it results in the following deformation

$$
f\left(x, y, t_{3}, t_{2}, t_{1}\right)=y^{3}-t_{2} x y^{2}-t_{1} t_{3} x^{2} y-t_{3}^{2} x^{3}+t_{1} x^{3} y+2 t_{3} x^{4}-x^{5} .
$$

This deformation satisfies that for certain values of $t_{1}, t_{2}, t_{3}$ with $t_{i} \neq t_{j}$ for $i \neq j$, the curve $C_{\underline{t}}$ given by the equation $f\left(x, y, t_{3}, t_{2}, t_{1}\right)=0$ has a unique ordinary singular point of multiplicity 3 at $(0,0)$ and a double point outside the origin. 
Following A'Campo's method, on the contrary, we have

$$
\bar{f}(x, y, t)=f(x, t, t, t)=y^{3}-t x y^{2}+t^{2} x^{2} y-t^{2} x^{3}+t x^{3} y-2 t x^{4}-x^{5}
$$

which, for arbitrary small $t$ has one real branch and two complex conjugated ones. This can be seen by extracting the homogeneous form of degree 3 of $\bar{f}(x, y, t)$, that is, $\bar{f}_{3}(x, y, t)=y^{3}-t x y^{2}+t^{2} x^{2} y-t^{2} x^{3}$ and deshomogenizing for $x \neq 0$. The polynomial $\bar{f}_{3}(1, y, t)=y^{3}-t y^{2}+t^{2} y-t^{2}$ has only one real root since the discriminant of $\frac{\partial \bar{f}_{3}}{\partial y}$ with respect to $y$ is $-8 t^{2} \leq 0$ for $t \in \mathbb{R}-\{0\}$.

In this section we will study deformations into nodes of the singularity types $y^{2 d-1}-x^{2 d+1}=0$. We will prove that A'Campo's method of blowing-ups and translations can be carried out in this case, but on parametric equations rather than on implicit equations. Another difference with A'Campo's method is the kind of substitutions used: $t_{1}=\beta_{1}(s)$; $t_{2}=\beta_{2}(s) ; \ldots ; t_{k}=\beta_{k}(s)$, where $\beta(s)$ is an analytic path, instead of $t_{1}=t_{2}=\ldots=t_{k}=t$.

Let $f(x, y)=y^{2 d-1}-x^{2 d+1} \in \mathbb{C}[x, y], d \geq 2$. Its zero locus, the plane curve $H=\left\{(x, y) \in \mathbb{C}^{2} ; f(x, y)=0\right\}$, has an isolated singularity at $(0,0)$. Let us consider the following parametrization of $H$

$$
\gamma_{0}=\left(\gamma_{01}, \gamma_{02}\right): I \longrightarrow \mathbb{R}^{2} ; \gamma_{0}(u)=(x, y)=\left(u^{2 d-1}, u^{2 d+1}\right)
$$

and a sequence of quadratic transformations $\left(\pi_{i}\right)_{i=1,2, \ldots, d+1}$ defined as follows:

$$
\begin{gathered}
\pi_{1}\left(x_{1}, y_{1}\right)=(x, y)=\left(x_{1}, x_{1} y_{1}\right) ; \\
\pi_{j}\left(x_{j}, y_{j}\right)=\left(x_{j-1}, y_{j-1}\right)=\left(x_{j} y_{j}, y_{j}\right), j=2,3, \ldots, d ; \\
\pi_{d+1}\left(x_{d+1}, y_{d+1}\right)=\left(x_{d}, y_{d}\right)=\left(x_{d+1}, x_{d+1} y_{d+1}\right)
\end{gathered}
$$

Performing these transformation successively on $\gamma_{0}$ one obtains: 


\begin{tabular}{|c|c|c|c|c|}
\hline$(x, y)$ & $=$ & $\left(u^{2 d-1}, u^{2 d+1}\right)$ & $\longleftrightarrow$ & $y^{2 d-1}-x^{2 d+1}=0$ \\
\hline$\left(x_{1}, y_{1}\right)$ & $\begin{array}{l}\uparrow \pi_{1} \\
= \\
\uparrow \pi_{2}\end{array}$ & $\left(u^{2 d-1}, u^{2}\right)$ & $\longleftrightarrow$ & $y_{1}^{2 d-1}-x_{1}^{2}=0$ \\
\hline$\left(x_{2}, y_{2}\right)$ & $\begin{array}{l}= \\
\uparrow \pi_{3}\end{array}$ & $\left(u^{2 d-3}, u^{2}\right)$ & $\longleftrightarrow$ & $y_{2}^{2 d-3}-x_{2}^{2}=0$ \\
\hline$\left(x_{3}, y_{3}\right)$ & $\begin{array}{l}= \\
\uparrow \pi_{4}\end{array}$ & $\left(u^{2 d-5}, u^{2}\right)$ & $\longleftrightarrow$ & $y_{3}^{2 d-5}-x_{3}^{2}=0$ \\
\hline & $\vdots$ & & & $\vdots$ \\
\hline$\left(x_{d}, y_{d}\right)$ & $\begin{array}{l}\uparrow \pi_{d} \\
= \\
\uparrow \pi_{d+1}\end{array}$ & $\left(u, u^{2}\right)$ & $\longleftrightarrow$ & $y_{d}-x_{d}^{2}=0$ \\
\hline,$\left.y_{d+1}\right)$ & $=$ & $(u, u)$ & $\longleftrightarrow$ & $y_{d+1}-x_{d+1}=0$ \\
\hline
\end{tabular}

Implicit equations of the non-exceptional components of the resolution are shown on the second column of the previous figure. Their sequence of multiplicities shows that there is one point of multiplicity $2 d-1$ and also $d-1$ points of multiplicity 2. After the process shown next, we will manage to obtain $d-1$ double points and one point of multiplicity $2 d-1$. This can be achieved performing the following translations and contractions on the last non-exceptional branch, where $t_{j} \in \mathbb{R}-\{0\}$, $j=1,2, \ldots, d+1$

$$
\begin{gathered}
\left(x_{d+1}, y_{d+1}\right)=(u, u) \stackrel{\tau_{1}}{\longrightarrow}\left(\bar{x}_{d+1}, \bar{y}_{d+1}\right)=\left(x_{d+1}, y_{d+1}+t_{1}\right)=\left(u, u+t_{1}\right) \\
\stackrel{\pi_{d+1}}{\longrightarrow}\left(x_{d}, y_{d}\right)=\left(u, u\left(u+t_{1}\right)\right) \stackrel{\tau_{2}}{\longrightarrow}\left(\bar{x}_{d}, \bar{y}_{d}\right)=\left(u, u\left(u+t_{1}\right)+t_{2}\right)=\left(u, \varphi_{1}\right) \\
\stackrel{\pi_{d}}{\longrightarrow}\left(x_{d-1}, y_{d-1}\right)=\left(u \varphi_{1}, \varphi_{1}\right) \stackrel{\tau_{3}}{\longrightarrow}\left(\bar{x}_{d-1}, \bar{y}_{d-1}\right)=\left(u \varphi_{1}, \varphi_{1}+t_{3}\right)= \\
=\left(u \varphi_{1}, \varphi_{2}\right) \stackrel{\pi_{d-1}}{\longrightarrow}\left(x_{d-2}, y_{d-2}\right)=\left(u \varphi_{1} \varphi_{2}, \varphi_{2}\right) \stackrel{\tau_{4}}{\longrightarrow}\left(\bar{x}_{d-2}, \bar{y}_{d-2}\right)= \\
=\left(u \varphi_{1} \varphi_{2}, \varphi_{2}+t_{4}\right)=\left(u \varphi_{1} \varphi_{2}, \varphi_{3}\right) \stackrel{\pi_{d-2}}{\longrightarrow} \ldots,
\end{gathered}
$$

where $\varphi_{1}$ denotes $u\left(u+t_{1}\right)+t_{2}, \varphi_{2}$ denotes $\varphi_{1}+t_{3}$, and so on $\varphi_{k}$ denotes $\varphi_{k-1}+t_{k+1}$ up until the one-to-last contraction $\pi_{2}$, 


$$
\ldots \stackrel{\pi_{2}}{\longrightarrow}\left(x_{1}, y_{1}\right)=\left(u \varphi_{1} \varphi_{2} \varphi_{3} \ldots \varphi_{d-1}, \varphi_{d-1}\right) .
$$

The graph of this last parametrization becomes

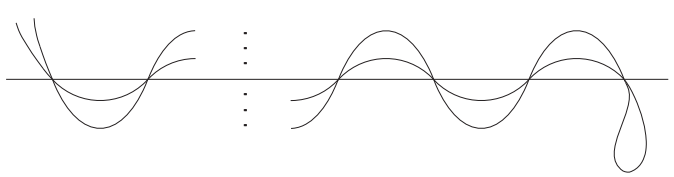

Note that the $d-1$ double points appear by considering both roots of $\varphi_{1}$ up until $\varphi_{d-1}$. Let $u^{k}$ be a root of $\varphi_{k}, k \in\{1,2, \ldots, d-1\}$; then one has

$$
\left(u^{k} \varphi_{1}\left(u^{k}\right) \varphi_{2}\left(u^{k}\right) \ldots \varphi_{d-1}\left(u^{k}\right), \varphi_{d-1}\left(u^{k}\right)\right)=\left(0, \sum_{j=d-k+1}^{d} t_{j}\right) .
$$

The last translation $\tau_{d+1}$ is performed in a direction normal to the $x_{1}$ axis, whereas the previous ones were performed in a direction normal to the $y_{j}$-axis. This last translation becomes

$$
\stackrel{\tau_{d+1}}{\longrightarrow}\left(\bar{x}_{1}, \bar{y}_{1}\right)=\left(x_{1}+t_{d+1}, y_{1}\right)=\left(u \varphi_{1} \varphi_{2} \varphi_{3} \ldots \varphi_{d-1}+t_{d+1}, \varphi_{d-1}\right)
$$

The graph, after this parametrization becomes

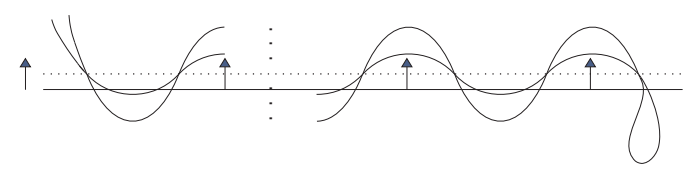

Finally, after contracting by $\pi_{1}$ we obtain

$\stackrel{\pi_{1}}{\longrightarrow}(x, y)=\left(u \varphi_{1} \varphi_{2} \varphi_{3} \ldots \varphi_{d-1}+t_{d+1}, \varphi_{d-1}\left(u \varphi_{1} \varphi_{2} \varphi_{3} \ldots \varphi_{d-1}+t_{d+1}\right)\right)$

which is the parametrization of a curve $C_{\underline{t}}$ denoted by:

$$
\gamma(u, \underline{t})=\gamma\left(u, t_{1}, t_{2}, \ldots, t_{d+1}\right)=(x, y) .
$$

Note that if $t_{1}=t_{2}=\ldots=t_{d+1}=0$ one has

$\varphi_{1}=\varphi_{2}=\varphi_{3}=\ldots=\varphi_{d-1}=u^{2}$. Therefore $\gamma(u, \underline{0})=\left(u^{2 d-1}, u^{2 d+1}\right)=$ 
$\gamma_{0}(u)$

Using the previous part, we can decide where the $d-1$ double points of the curve $\gamma(u, \underline{t})$ lay. In order to do that, let us consider the curve obtained right before the last contraction,

$$
\gamma^{*}(u, \underline{t})=\left(x_{1}, y_{1}\right)=\left(u \varphi_{1} \varphi_{2} \varphi_{3} \ldots \varphi_{d-1}+t_{d+1}, \varphi_{d-1}\right) .
$$

Let us assume that $u_{1}^{k}$ and $u_{2}^{k}$ are the two roots of $\varphi_{k} ; k \in\{1,2, \ldots, d-1\}$. Hence, for each

$$
\begin{aligned}
& k \in\{1,2, \ldots, d-2\}, \text { one has } \gamma^{*}\left(u_{1}^{k}, \underline{t}\right)=\gamma^{*}\left(u_{2}^{k}, \underline{t}\right)=\left(t_{d+1}, \sum_{j=k+2}^{d} t_{j}\right) \\
& \text { and for } \quad k=d-1, \text { one has } \gamma^{*}\left(u_{1}^{d-1}, \underline{t}\right)=\gamma^{*}\left(u_{2}^{d-1}, \underline{t}\right)=\left(t_{d+1}, 0\right) .
\end{aligned}
$$

Let us find out under what conditions the equations $\varphi_{j}, j=1,2, \ldots, d-$ 1 , have two distinct real roots.

Since $\varphi_{1}=u\left(u+t_{1}\right)+t_{2}=u^{2}+t_{1} u+t_{2}$, it is enough to check that $t_{1}^{2}-4 t_{2}>0$, or equivalently, $t_{2}<\frac{1}{4} t_{1}^{2}$. On the other hand, since $\varphi_{j}=\varphi_{1}+\sum_{i=3}^{j+1} t_{i}=u^{2}+t_{1} u+\sum_{i=2}^{j+1} t_{i}$ for each $j=2,3, \ldots, d-1$, it is enough to verify that $t_{1}^{2}-4 \sum_{i=2}^{j+1} t_{i}>0$, that is, $t_{j+1}<\frac{1}{4} t_{1}^{2}-\sum_{i=2}^{j} t_{i}$.

Remark. Note that each double point $P_{k}$ can be obtained after contracting by $\pi_{d-k+2}$. The following condition $t_{k}<\frac{1}{4} t_{1}^{2}-\sum_{j=2}^{k-1} t_{j}$ implies that $\pi_{d-k+2}\left(P_{k}\right)$ are two distinct points. Since $\pi_{d-k+2}$ is a quadratic transformation, the points in $\pi_{d-k+2}\left(P_{k}\right)$ correspond to tangents at $P_{k}$, and hence, there are two distinct tangents at $P_{k}$, therefore it is a node.

Proposition 4.1. In the previous conditions, the polynomial $u \varphi_{1} \varphi_{2} \varphi_{3}$ $\ldots \varphi_{d-1}+t_{d+1}$ has $2 d-1$ real roots for an arbitrary small $t_{d+1} \in \mathbb{R}-\{0\}$.

Proof. Let us consider the parametrization right before the last translation, 


$$
\theta=\left(\theta_{1}, \theta_{2}\right)=\left(u \varphi_{1} \varphi_{2} \varphi_{3} \ldots \varphi_{d-1}, \varphi_{d-1}\right)
$$

As we mentioned before, the image of $\theta$ has $d-1$ nodes at $P_{1}, P_{2}, \ldots, P_{d-1}$ whose tangents are all different from each other and also different from the last exceptional component $E_{1}$, which is the $y_{1}$-axis. Consider $P_{k}=\theta\left(u_{j}^{k}\right)(j=1,2)$, then the tangent vector becomes

$$
\theta^{\prime}\left(u_{j}^{k}\right)=\left(\theta_{1}^{\prime}\left(u_{j}^{k}\right), \theta_{2}^{\prime}\left(u_{j}^{k}\right)\right) .
$$

The exceptional component $E_{1}\left(x_{1}=0\right)$ is tangent at $P_{k}$ if and only if $\theta_{1}^{\prime}\left(u_{j}^{k}\right)=0$. Hence, by construction $\theta_{1}(u)=u \varphi_{1} \varphi_{2} \varphi_{3} \ldots \varphi_{d-1}$ has $2 d-1$ distinct roots, that is, $\theta_{1}(u)$ and $\theta_{1}^{\prime}(u)$ have no common roots.

Since $E_{1}$ is transversal to $\operatorname{Im}(\theta)$ at every $P_{k}$, after performing the translation $\bar{x}_{1}=x_{1}+t_{d+1}$, the axis $\bar{x}_{1}=0$ intersects the curve in two points close to $P_{k}$ and different from each other if $t_{d+1}$ is sufficiently small. This can be shown by a direct application of Theorem (2.1).



Note also that $\theta_{1}(u)+t_{d+1}$ has $2 d-1$ real roots if $t_{d+1}$ verifies $\left|t_{d+1}\right|<h\left(t_{1}, t_{2}, \ldots, t_{d}\right)$, where $h\left(t_{1}, t_{2}, \ldots, t_{d}\right)$ is the minimum of $\left|x_{1}\right|$ on the extreme values of the curves.

Proposition 4.2. The curve

$$
\gamma(u, \underline{t})=\left(u \varphi_{1} \varphi_{2} \varphi_{3} \ldots \varphi_{d-1}+t_{d+1}, \varphi_{d-1}\left(u \varphi_{1} \varphi_{2} \varphi_{3} \ldots \varphi_{d-1}+t_{d+1}\right)\right),
$$


where $t_{k}<\frac{1}{4} t_{1}^{2}-\sum_{j=2}^{k-1} t_{j},(k=2,3, \ldots, d)$ and $t_{d+1}$ are arbitrary small, has an ordinary double point of multiplicity $2 d-1$ and also $d-1$ distinct double points.

Proof. It is enough to apply Proposition (4.1), where $\operatorname{Im}(\theta) \cap E_{1}$ are $2 d-1$ distinct points. After contructing by $\pi_{1}, 2 d-1$ branches are produced with different tangents.

Proposition 4.3. There exists an analytic curve $\beta:(-\varepsilon, \varepsilon) \longrightarrow \mathbb{R}^{d+1}$ such that $\underline{t}=\beta(t)$ and, satisfying that the image of the parametrization $\gamma(u, \beta(t))$ has one ordinary point of multiplicity $2 d-1$ and also $d-1$ distinct ordinary double points.

Proof. Let

$U=\left\{\bar{t} \in \mathbb{R}^{d+1} ; t_{k}<\frac{1}{4} t_{1}^{2}-\sum_{j=2}^{k-1} t_{j},(k=2,3, \ldots, d) a n d t_{d+1}<h\left(t_{1}, \ldots, t_{d}\right)\right\}$.

Let us consider the sequence $\left(t_{1 n}\right)$ such that $t_{1 n} \longrightarrow 0$. We can construct the following sequences $\left(t_{k n}\right),(k=2,3, \ldots, d)$ such that $t_{k n}<\frac{1}{4} t_{1 n}^{2}-$ $\sum_{j=2}^{k-1} t_{j n}$ and $\left(t_{(d+1) n}\right)$ with $t_{(d+1) n}<h\left(t_{1 n}, t_{2 n}, \ldots, t_{d n}\right)$. It is obvious that all the so-constructed sequences converge to 0 . Hence,

$$
\underline{t}_{n}=\left(t_{1 n}, t_{2 n}, \ldots, t_{(d+1) n}\right) \longrightarrow \underline{0}_{n} \in U .
$$

By Milnor's Curve Selection Lemma, [7], there exists an analytic curve $\rho:[0, \varepsilon) \longrightarrow \mathbb{R}^{d+1}$ such that $\rho(0)=\underline{0}$ and $\rho(t) \in U$ for $t>0$. Performing the following change $t=t^{2} ; \bar{\rho}:(-\varepsilon, \varepsilon) \longrightarrow[0, \varepsilon), \bar{\rho}(t)=t^{2}$, one has $\beta=\rho \circ \bar{\rho}$ and $\beta((-\varepsilon, \varepsilon)-\{0\}) \subset U$.

Theorem 4.1. Let $f(x, y)=y^{2 d-1}-x^{2 d+1} \in \mathbb{C}[x, y], d \geq 2$, be a polynomial and let $\gamma(u, t)=\gamma(u, \beta(t))$ be the parametrization described in the previous proposition. The function

$$
f(x, y ; t)=\operatorname{Res}_{u}\left(x-\gamma_{1}(u, t), y-\gamma_{2}(u, t)\right) \in \mathbb{R}[x, y]\{t\}
$$

is a deformation of $f(x, y)$, and the plane curve $C_{t}=\left\{(x, y) \in \mathbb{C}^{2} ; f(x, y ; t)\right.$ $=0\} \subset \mathbb{C}^{2}$ has a unique ordinary singular point of multiplicity $2 d-1$ at the origin, as well as $d-1$ ordinary double points. 
Proof. This proof is similar to the one of Theorem (2.3) and Proposition (3.4), since $\gamma_{1}(u, t)$ and $\gamma_{2}(u, t)$ are of the form:

$$
\begin{aligned}
& \gamma_{1}(u, t)=u^{2 d-1}+a_{1} u^{2 d-2}+a_{2} u^{2 d-3}+\ldots+a_{2 d-2} u+a_{2 d-1} \\
& \gamma_{2}(u, t)=u^{2 d+1}+b_{1} u^{2 d}+b_{2} u^{2 d-1}+\ldots+b_{2 d-1} u^{2}+b_{2 d} u+b_{2 d+1}
\end{aligned}
$$

where the coefficients $a_{i}$ and $b_{j}, i=1,2, \ldots, 2 d-1 ; j=1,2, \ldots, 2 d+1$ are analytic functions in $t,\left(a_{i}=a_{i}(t) ; b_{j}=b_{j}(t)\right)$ without degree zero terms in $t$. On the other hand, at $t=0$ one has $f(x, y ; 0)=$

$$
=\operatorname{Res}_{u}\left(x-\gamma_{1}(u, 0), y-\gamma_{2}(u, 0)\right)=\operatorname{Res}_{u}\left(x-u^{2 d-1}, y-u^{2 d+1}\right)=
$$

$=y^{2 d-1}-x^{2 d+1}=f(x, y)$. Therefore, the result follows from Theorem $(2.3)$.

Let $f(x, y ; t)$ be the deformation of $f(x, y)=y^{2 d-1}-x^{2 d+1}$ described in the previous Theorem and let $\gamma(u, t)$ be the parametrization of $C_{t}$ above considered. For every $t \neq 0, \gamma\left(e_{j}(t), t\right)=\underline{0}$. Performing the change of variable $t=\tilde{t}^{n}$ it is possible to obtain that $e_{j}(t)$ are analytic functions in $\tilde{t}$. Moreover,

Proposition 4.4. In the previous conditions, there exists a natural number $n \in \mathbb{N}$ and $2 d-1$ analytic functions $e_{1}(\tilde{t}), e_{2}(\tilde{t}), \ldots, e_{2 d-1}(\tilde{t}) \in$ $\mathbb{R}\{t\}$ such that, for any $|\tilde{t}|$ sufficiently small, one has $\gamma\left(e_{j}(\tilde{t}), \tilde{t}^{n}\right)=\underline{0}$.

Proof. The curve $H=\{(u, t) ; \gamma(u, t)=(0,0)\}$ is analytic, since, by construction of $\gamma(u, t), \gamma(u, t) \in \mathbb{R}[u]\{t\}$.

For any $t \neq 0$, the values of $u$ that satisfy $\gamma(u, t)=\underline{0}$ are the roots of

$$
\gamma_{1}^{*}(u, t)=\theta_{1}(u, t)+t_{d+1}(t)=u \varphi_{1} \varphi_{2} \ldots \varphi_{d-1}+t_{d+1}(t) \in \mathbb{R}[u]\{t\}
$$

where $\gamma_{1}^{*}(u, t)$ is a polynomial of degree $2 d-1$ in $u$. In other words, the projection $\pi: H_{\mathbb{C}} \longrightarrow E_{t}$ is a $2 d-1$-covering -for each value of $t$ arbitrarily small there are $2 d-1$ solutions close to zero. That is, there exists $\Delta(0)$ (a complex ball) such that for any $t$, there exist $u_{1}(t), u_{2}(t), \ldots, u_{2 d-1}(t) \in \mathbb{C}$ verifying $\gamma\left(u_{j}(t), t\right)=(0,0)$.

By the Local Parametrization Theorem, the above is equivalent to the fact that $\gamma(u, t)$ is regular and has order $2 d-1$ in $u$. Hence, by Weierstrass Preparation Theorem, there exists a unit $l(u, t)(l(0,0) \neq 0)$, such that 


$$
\gamma(u, t) l(u, t)=u^{2 d-1}+\sum_{j=1}^{2 d-1} A_{j}(t) u^{2 d-1-j}=Q(u, t)
$$

By Newton-Puiseux Theorem, the $2 d-1$ roots of $Q(u, t)$ are series in $t^{\frac{1}{n}}$ for a certain $n, e_{j}\left(t^{\frac{1}{n}}\right)$. Therefore $Q(u, t)=\prod_{j=1}^{2 d-1}\left(u-e_{j}\left(t^{\frac{1}{n}}\right)\right)$. If we consider the change of variable $t=\tilde{t}^{n}$, one has

$$
Q\left(u, \tilde{t}^{n}\right)=\prod_{j=1}^{2 d-1}\left(u-e_{j}(\tilde{t})\right)=\gamma\left(u, \tilde{t}^{n}\right) l\left(u, \tilde{t}^{n}\right)
$$

That is, $\gamma\left(e_{j}\left(\tilde{t}, \tilde{t}^{n}\right)=(0,0)\right.$, for any $j=1,2, \ldots, 2 d-1$. Note that $e_{j} \in \mathbb{C}\{\tilde{t}\}$, but if $t$ is real and sufficiently small, then $e_{j}(\tilde{t})$ are all real, since they are the roots of $u \varphi_{1} \varphi_{2} \ldots \varphi_{d-1}+t_{d+1}(t)$. Therefore also the series $e_{j}(\tilde{t}) \in \mathbb{R}\{\tilde{t}\}$.

Remark. Let $\tilde{\gamma}(u, \tilde{t})=\gamma\left(u, \tilde{t}^{n}\right)$. From now on, we will use $\tilde{\gamma}$ and $\tilde{t}$, but for the sake of simplicity we will denote them by $\gamma$ and $t$ respectively. Hence, $\gamma\left(e_{j}(t), t\right)=(0,0), j=1,2, \ldots, d-1$. Therefore, $(0,0)$ is an ordinary singular point of multiplicity $2 d-1$ of the curve $\operatorname{Im}(\gamma(u, t))=$ $C_{t}$.

Let $f(x, y ; t) \in \mathbb{C}[x, y]\{t\}$ and let $f(x, y ; t)=0$ be the implicit equation of $C_{t}$ obtained by Theorem (4.1). By the above discussion, the initial form of $f(x, y ; t)$ as a polynomial in the variables $x$ and $y$ has degree $2 d-1$. We will denote such an initial form by $f_{2 d-1}(x, y ; t)$.

By construction, $f_{2 d-1}(x, y ; 0)=y^{2 d-1}$. This means, that

$$
f_{2 d-1}(x, y ; t)=y^{2 d-1}+\sum_{j=1}^{2 d-1} B_{j}(t) x^{j} y^{2 d-1-j}
$$

Following step by step the proof of Proposition (4.4), it is clear that, after performing the following change $t=\tilde{t}^{n}$, one obtains

$$
\tilde{f}_{2 d-1}(x, y ; \tilde{t})=f_{2 d-1}\left(x, y ; \tilde{t}^{n}\right)=\prod_{j=1}^{2 d-1}\left(y-\alpha_{j}(\tilde{t}) x\right)
$$

Once again, for the sake of simplicity, we will denote $\tilde{f}_{d-1}$ (resp. $\tilde{t}$ ) by $f_{d-1}$ (resp. $t$ ). Thus, the tangent lines at the origin are $l_{j} \equiv \alpha_{j}(t) x-y=$ $0, j=1,2, \ldots, 2 d-1$. 
For any $j \neq k$ we will define $e_{j}(t)-e_{k}(t)=t^{n_{j k}} \varepsilon_{j k}(t)$, where $\varepsilon_{j k}(0) \neq$ 0 . Let $n_{j}=\sum_{k=1}^{2 d-1} n_{j k}$ and $n=\max \left\{n_{j} ; 1 \leq j \leq 2 d-1\right\}$.

$$
a \neq j
$$

Let $\mathbf{v}^{j}=\left(v_{1}^{j} t^{n+1}, v_{2}^{j} t^{n+1}\right) \in \mathbb{R}^{2}, j=1,2, \ldots, 2 d-1$; be vectors such that the translated lines $\bar{l}_{j}=l_{j}+\mathbf{v}^{j}$ intersect in distinct points. Therefore, $\bar{l}_{j} \equiv \alpha_{j}(t)\left(x-v_{1}^{j} t^{n+1}\right)-\left(y-v_{2}^{j} t^{n+1}\right), j=1,2, \ldots, 2 d-1$.

Let $p_{t}(u)=\left(p_{1}(u), p_{2}(u)\right)$, where $p_{1}(u), p_{2}(u) \in \mathbb{R}[u]$ are polynomials such that $p_{t}\left(e_{j}(t)\right)=\left(v_{1}^{j} t^{n}, v_{2}^{j} t^{n}\right), j=1,2, \ldots, 2 d-1$. Such polynomials exist for large enough degrees.

Proposition 4.5. Under the previous conditions, let

$$
L_{j}(u, t)=\prod_{\substack{k=1 \\ k \neq j}}^{2 d-1} \frac{\left(u-e_{k}(t)\right)}{\varepsilon_{j k}(t)} \quad, \quad j=1,2, \ldots, 2 d-1 .
$$

If we define

$$
p_{1}(u, t)=\sum_{j=1}^{2 d-1} v_{1}^{j} t^{n-n_{j}} L_{j}(u, t) \quad \text { and } \quad p_{2}(u, t)=\sum_{j=1}^{2 d-1} v_{2}^{j} t^{n-n_{j}} L_{j}(u, t),
$$

one has $p_{1}(u), p_{2}(u) \in \mathbb{R}[u], p_{1}\left(e_{j}(t), t\right)=v_{1}^{j} t^{n}$ and $p_{2}\left(e_{j}(t), t\right)=v_{2}^{j} t^{n}$

Proof. The result follows using the Lagrange polynomial for interpolation and the fact that $\varepsilon_{j k} \neq 0\left(\right.$ since $\left.n \geq n_{j}\right)$.

Proposition 4.6. Let $\bar{\gamma}(u, b, t)=\gamma(u, t)+b t p_{t}(u), \quad b \in \mathbb{R}-\{0\}$.

For any $b$ and $t$ sufficiently small, the curve $\bar{\gamma}(u, b, t)$ is a partition at the origin of $2 d-1$ branches intersecting generically. Moreover, it also has $d-1$ double points inherited from $\gamma(u, t)$.

Proof. It is based on Theorem (2.1) and its proof is similar to the one of Proposition (3.2). In this case the intersection point of $\bar{l}_{i}$ and $\bar{l}_{k}$ has coordinates

$$
\begin{aligned}
& P_{i k}^{1}(t)=\left(\alpha_{i}(t) v_{1}^{i}-\alpha_{k}(t) v_{1}^{k}-\left(v_{2}^{i}-v_{2}^{k}\right)\right) \frac{t^{n+1-n_{i k}}}{\varepsilon_{i k}(t)} \\
& P_{i k}^{2}(t)=\left(\alpha_{i}(t) \alpha_{k}(t)\left(v_{1}^{i}-v_{1}^{k}\right)+\alpha_{i}(t) v_{2}^{k}-\alpha_{k}(t) v_{2}^{i}\right) \frac{t^{n+1-n_{i k}}}{\varepsilon_{i k}(t)}
\end{aligned}
$$


It is obvious that one can choose $v_{1}^{i}, v_{2}^{i}, v_{1}^{k}$ and $v_{2}^{k}$ so that the first term in the Taylor expansion of $P_{i k}(t)=\left(P_{i k}^{1}(t), P_{i k}^{2}(t)\right)$ is different for each $i$, $k(i \neq k)$. In other words, if $t$ is sufficiently small, the intersection points of $\bar{l}_{i}$ and $\bar{l}_{k}$ are different. The result follows as in Proposition (2.2).

Proposition 4.7. Under the hypothesis of the previous proposition (4.6) the arrangement of the $2 d-1$ branches of $\bar{\gamma}(u, b, t)$ produces a partition with the same real combinatorics as the $2 d-1$ lines $\bar{l}_{j}, j=1,2, \ldots 2 d-1$.

Proof. It is direct consequence of Theorem (2.2) under the hypothesis of Proposition (4.6).

Proposition 4.8. Under the previous conditions, let

$$
\bar{\gamma}(u, b, t)=\gamma(u, t)+b t p_{t}(u)=\left(\bar{\gamma}_{t}^{1}(u, b), \bar{\gamma}_{t}^{2}(u, b)\right)
$$

be a parametrization, where

$$
\bar{\gamma}_{t}^{1}(u, b)=\gamma_{1}(u, t)+b t p_{1}(u, t) \quad, \quad \bar{\gamma}_{t}^{2}(u, b)=\gamma_{2}(u, t)+b t p_{2}(u, t) .
$$

The function $\bar{f}(x, y ; b, t)=\operatorname{Res}_{u}\left(\bar{\gamma}_{t}^{1}(u, b)-x, \bar{\gamma}_{t}^{2}(u, b)-y\right)$ is a polynomial deformation of degree $2 d+1$ of $f(x, y)=y^{2 d-1}-x^{2 d+1}$.

Proof. The proof is similar to the one of Theorem (2.3), (4.1) and Proposition (3.4). Let us recall that

$$
\begin{aligned}
& \bar{\gamma}_{t}^{1}(u, b)=u \varphi_{1} \varphi_{2} \varphi_{3} \ldots \varphi_{d-1}+t_{d+1}(t)+b t p_{1}(u, t) \\
& \bar{\gamma}_{t}^{2}(u, b)=\varphi_{d-1}\left(u \varphi_{1} \varphi_{2} \varphi_{3} \ldots \varphi_{d-1}+t_{d+1}(t)\right)+b t p_{2}(u, t),
\end{aligned}
$$

Therefore

$$
\begin{aligned}
& \bar{\gamma}_{t}^{1}(u, b)=u^{2 d-1}+a_{1} u^{2 d-2}+a_{2} u^{2 d-3}+\ldots+a_{2 d-2} u+a_{2 d-1} \\
& \bar{\gamma}_{t}^{2}(u, b)=u^{2 d+1}+b_{1} u^{2 d}+b_{2} u^{2 d-1}+\ldots+b_{2 d-1} u^{2}+b_{2 d} u+b_{2 d+1}
\end{aligned}
$$

where the coefficients $a_{i}$ and $b_{j}, i=1,2, \ldots, 2 d-1 ; j=1,2, \ldots, 2 d+1$ are analytic functions in $t,\left(a_{i}=a_{i}(t) ; b_{j}=b_{j}(t)\right)$ with no degree zero term in $t$. Hence, the result follows by Theorem (2.3).

Let $U=\left\{(b, t) \in \Delta \subset \mathbb{C}^{2}\right.$, then $\bar{\gamma}(u, b, t)$ has $\left(\begin{array}{c}2 d-1 \\ 2\end{array}\right)$ distinct double points (in addition to the $d-1$ inherited from $\gamma(u, t)$ ) $\}$.

Theorem 4.2. Under the above conditions, there exists $D(b, t) \in \mathbb{R}\{b, t\}$ such that the set $U \supset\{(b, t) \in \Delta ; D(b, t) \neq 0\}$, is a dense Zariski 
open set, and therefore there exists an analytic curve $\beta: J \longrightarrow \Delta$; $\beta(s)=(b(s), t(s))$ such that $\beta(J-\{0\}) \subset U$. Moreover, $\tilde{\gamma}(u, s)=$ $\bar{\gamma}(u, b(s), t(s))=\bar{\gamma}(u, \beta(s))$ verifies that, for any $s \neq 0$, the curve $\tilde{\gamma}(u, s)$ has $\left(\begin{array}{c}2 d-1 \\ 2\end{array}\right)$ distinct double points, in addition to the $d-1$ points inherited from $\gamma(u, t(s))$.

Proof. The proof follows step by step the one in Theorem (3.1).

Theorem 4.3. Let $f(x, y)=y^{2 d-1}-x^{2 d+1} \in \mathbb{C}[x, y]$ be a polynomial with $d \geq 2$. Then there exist deformations $\bar{f}(x, y ; s)$ of $f(x, y)$, of degree $2 d+1$, such that, for any $s \in \mathbb{R}-\{0\}$ sufficiently small, the plane curve $\bar{C}_{s} \equiv \bar{f}(x, y ; s)=0$ has $\left(\begin{array}{c}2 d-1 \\ 2\end{array}\right)+d-1$ distinct real double points near the origin.

Proof. It is a straightforward consequence of the previous propositions.


$C_{t}$ and $\bar{C}_{t}$ (Case $d=2$ )
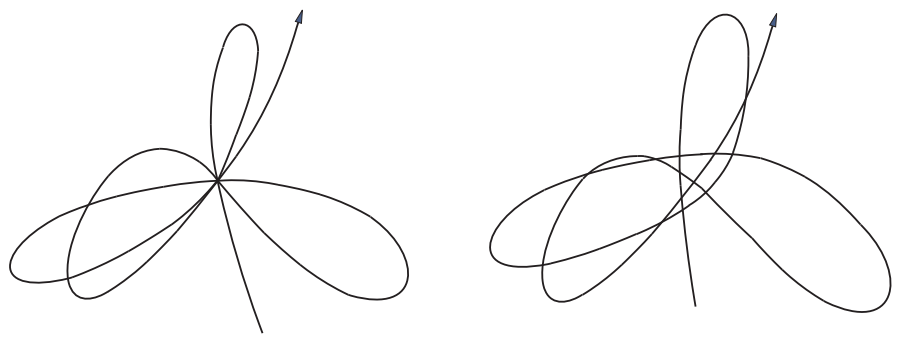

$C_{t}$ and $\bar{C}_{t}$ (Case $d=3$ ) 


\section{References}

[1] A'Campo, N., Le groupe de monodromie du déploiment des singularites isolées de courbes planes I, Math. Ann. 213. Springer-Verlag (1.975), 1-32.

[2] Benedetti, R., Risler, J.J., Real algebraic and semi-algebraic sets, Actualités Mathématiques. Hermann, Editeurs., 1.990.

[3] Geddes, K., Czapor, S., Labahn, G., Algoritms in Computer Algebra, Kluwer A. P. 1.992.

[4] Fulton, W., Algebraic curves, Benjamín, 1.969.

[5] González-Ramírez, J.A., Contribución al estudio de las deformaciones de singularidades reales, Tesis Doctoral. Universidad Nacional de Educación a Distancia, 1.996.

[6] Gusein-Zade, S.M., Dynkin diagrams for singularities of functions of two variables, Funktsionalnyi Analiz i ego Prilozheniya 8/4 (engl. Übers. in Functional Analysis and its Applications.) (1.974), 23-30.

[7] Milnor, J., Singular points of complex hypersurfaces, Ann of Math. 61. Princenton Univ. Press, 1.968.

[8] O'Shea, D., Topologically trivial deformations of isolated quasihomogeneous hipersurface singularities are equimultiple, Proc. A.M.S. 100, (1.987), 260-262.

[9] Teissier, B., The Hunting of Invariants in the Geometry of Discriminants, Real and Complex Singularities, Oslo 1.976. Sijthoff and Noordhoff international publeshers, 1.977 .

Centro Asociado a la

Universidad Nacional de Educación a Distancia

Ceuta

Spain

E-mail: jglezrez@wanadoo.es

Recibido: 15 de Marzo de 1999

Revisado: 30 de Mayo de 2001 